Verdoux, H., Lagnaoul, R. \& Bégaud, B. (2004) is benzodiazepine use a risk factor for cognitive decline and dementia? A literature review of epidemiological studies. Psychological Medicine, 35, I-9.

H. Verdoux, B. Bégaud Université Victor Segalen Bordeaux 2, Hôpital Charles Perrens, 121 rue de la Béchade, 33076 Bordeaux Cedex, France. E-mail: helene.verdoux@ipso.u-bordeaux2.fr

\section{Urbanisation and psychosis}

A recent study of urbanisation and incidence of psychosis was based on data from the excellent Swedish population registers (Sundquist et al, 2004). Unfortunately, there are methodological problems with the study.

Sundquist et al argue that the prospective nature of their data allowed them to calculate incidence rates of psychoses rather than prevalence rates. However, their study confuses readmission and incidence rates. In incidence studies, the event of interest is new occurrence of a specified disease (see e.g. Clayton \& Hills, 1993). Sundquist et al's cohort consists of people aged 25-64 years in 1996 (i.e. born 1932-1971). They analyse the data as incident data although they do not have this information. For persons who enter the study in old age, they claim that when admitted during the study period 1997-1999 this is the first admission ever of these people. They do, however, exclude people admitted 1992-1996, but this only partly solves this problem (e.g. for people born in 1932 in their study, the earliest possible age at onset of psychosis is 60 years). Let us say the earliest age at onset of psychosis is 25 years, the authors lack information on first admission for psychosis among people born 1966 or earlier. Roughly, they do not have information on first admission for psychosis among people born $1932-1966$, about $85 \%$ of the study population. If the earliest age of first admission for psychosis is 15 years (Pedersen $\&$ Mortensen, 2001), the authors lack information on first admission for all people. This severely violates the assumptions of an incidence study (Breslow \& Day, 1987), and the results may be extremely biased.

Sundquist et al measure urbanisation as the place where cohort members lived around 1996. Using this measure of urbanisation - in combination with the problems mentioned above - implies problems with causality; is the disease a result of the urbanisation or is the urbanisation a result of the disease?
Based on these methodological errors, it is difficult to interpret their findings. However, some possible biases include selective migration of people with psychosis after disease onset, and urban-rural differences in the readmission rates for people with psychosis.

Breslow, N. E. \& Day, N. E. (1987) Statistical Methods in Cancer Research. Vol. II: The Design and Analysis of Cohort Studies. IARC Scientific Publications No. 82. Lyon: International Agency for Research on Cancer.

Clayton, D. \& Hills, M. (1993) Statistical Models in Epidemiology. Oxford: Oxford University Press.

Pedersen, C. B. \& Mortensen, P. B. (200I) Family history, place and season of birth as risk factors for schizophrenia in Denmark: a replication and reanalysis. British Journal of Psychiatry, 179, 46-52.

Sundquist, K., Frank, G. \& Sundquist, J. (2004)

Urbanisation and incidence of psychosis and depression: follow-up study of 4.4 million women and men in Sweden. British Journal of Psychiatry, 184, 293-298.

C. B. Pedersen, E. Agerbo National Centre for Register-Based Research, University of Aarhus, Taasingegade I, 8000 Aarhus C, Denmark. E-mail: cbp@ncrr.dk

Authors' reply: We agree that in strict terms the definition of incidence rates is in accordance with the definition described by for example Clayton \& Hills (1993) and Last (1998).

However, we disagree when Pedersen \& Agerbo state that we claim 'this is the first admission ever of these people'. Nowhere in the article have we used the phrase 'first admission ever'. In the Method we clearly described that the 4.4 million women and men were followed from 1 January 1997 until 31 December 1999 for first admission to hospital for treatment of psychosis or depression (i.e. during the study period). Individuals with previous hospital admissions for treatment of psychosis or depression from 1992 to 1996 were excluded (i.e. the 'wash-out' period was 5 years). We calculated the urbanisation measure for the year 1996 (i.e. the year when we defined our study population).

Pedersen \& Agerbo also say that our article implies problems with causality. However, we did not claim that we have solved the causality problem. In addition, we discussed that we were unable to adjust our results for selective migration (i.e. migration from urban to rural areas). However, we did adjust our model for migration between neighbourhoods and the results remained almost unaltered.
We agree that urban-rural differences in admission rates could exist if, for example, the distribution of psychiatric beds differed between urban and rural areas. We checked this possible bias and found that the number of psychiatric beds per 1000 inhabitants differed very little across Sweden.

To sum up, our large-scale study sheds new light on the inconsistencies in previous research since we used the comprehensive Swedish registers and adjusted our results for several possible confounders. In addition, the urbanisation measure was calculated as actual numbers of inhabitants per area unit, which should reflect the level of urbanisation in the most appropriate way.

Clayton, D. \& Hills, M. (1993) Statistical Models in Epidemiology. Oxford: Oxford University Press.

Last, J. M. (1998) A Dictionary of Epidemiology (2nd edn). Oxford: Oxford University Press.

K. Sundquist, G. Frank, J. Sundquist Family Medicine Stockholm, Karolinska Institutet, Alfred Noblesallé 12, SE-14I 83 Huddinge, Sweden. E-mail: Kristina.Sundquist@klihvet.ki.se

\section{Anthropology and psychiatry}

From the perspective of anthropological psychiatry, Harland et al (2004) suggest that the social change experienced by migrants may predispose to schizophrenia. The same may apply to other forms of social change, such as those changes which lead to revitalisation phenomena and new religious movements. Psychiatric anthropologists who have studied new religious movements have pointed out that new forms of belief system may originate in the hallucinatory or delusional experience of a single individual (Littlewood, 1984). Wallace (1956) applied the term 'mazeway resynthesis' to the change in belief system which occurs in prophets, the mazeway being to the individual what culture is to society, so that the prophet awakes to a new reality which he or she then tries to impart to followers; if successful, the prophet becomes the leader of a new religious movement; otherwise, he or she is alienated from the parent group and is likely to be labelled as mentally ill. Foulks (1977) compared the prophet to the patient with schizophrenia, and noted that they shared not only the capacity to undergo a radical change in belief system but also various 'premorbid' features. 